\title{
Effect of the Velocity Second Slip Boundary Condition on the Peristaltic Flow of Nanofluids in an Asymmetric Channel: Exact Solution
}

\author{
Emad H. Aly ${ }^{1,2}$ and Abdelhalim Ebaid ${ }^{3}$ \\ ${ }^{1}$ Department of Mathematics, Faculty of Science, King Abdulaziz University, Jeddah 21589, Saudi Arabia \\ ${ }^{2}$ Department of Mathematics, Faculty of Education, Ain Shams University, Roxy, Cairo 11757, Egypt \\ ${ }^{3}$ Department of Mathematics, Faculty of Science, University of Tabuk, Tabuk 71491, Saudi Arabia \\ Correspondence should be addressed to Emad H. Aly; efarag@kau.edu.sa and Abdelhalim Ebaid; aebaid@ut.edu.sa
}

Received 10 October 2013; Accepted 5 February 2014; Published 23 March 2014

Academic Editor: Abdon Atangana

Copyright (C) 2014 E. H. Aly and A. Ebaid. This is an open access article distributed under the Creative Commons Attribution License, which permits unrestricted use, distribution, and reproduction in any medium, provided the original work is properly cited.

\begin{abstract}
The problem of peristaltic nanofluid flow in an asymmetric channel in the presence of the second-order slip boundary condition was investigated in this paper. To the best of the authors' knowledge, this parameter was here incorporated for the first time in such field of a peristaltic flow. The system governing the current flow was found as a set of nonlinear partial differential equations in the stream function, pressure gradient, nanoparticle concentration, and temperature distribution. Therefore, this system has been successfully solved exactly via a very effective procedure. These exact solutions were then proved to reduce to well-known results in the absence of second slip which were published very recently in the literature. Effect of the second slip parameter on the present physical parameters was discussed through graphs and it was found that this type of slip is a very important one to predict the investigated physical model. Moreover, the variation of many physical parameters such as amplitudes of the lower and upper waves, phase difference on the temperature distribution, nanoparticle concentration, pressure rise, velocity, and pressure gradient were also discussed. Finally, the present results may be viewed as an optimal choice for their dependence on the exact solutions which are obtained due to the highly complex nonlinear system.
\end{abstract}

\section{Introduction}

Peristalsis is an interest subject and has recently attracted much attention due to its importance in engineering and medical applications. In human body, peristalsis is found in the swallowing food through the esophagus, chyme motion in the gastrointestinal tract, vasomotion of small blood vessels such as venules, capillaries and arterioles, urine transport from kidney to bladder, and intrauterine fluid flow within the uterine cavity. Various experimental and theoretical studies have been conducted to understand peristaltic flow in asymmetric channel or an axisymmetric tube; see [17]. According to De Vries et al. [8], they observed that myometrial contractions are peristaltic-type motion and therefore these contractions of the uterine wall may occur in both symmetric and asymmetric directions. Due to the importance of this field of research, a great effort was devoted to study this type of flow for Newtonian and non-Newtonian fluids in an asymmetric channel [9-17].

Recently, a few papers have been published in the field of nanofluid flows under the peristaltic action [18-23]. Nanofluids usually lead to the enhancement of the thermal conductivity of the base fluid [24]. The concept of nanofluids, analogous to that of nanoparticles, may be initiated by Choi et al. [25]. The fact that nanofluids have higher thermal conductivity than the other heat transfer fluids because of their nanostructure has attracted many engineers and theoretical scientists to investigate their behavior. This higher thermal conductivity may be very useful in the treatment of tumors by injecting the blood vessel nearest to the tumor with magnetic nanoparticles along with placing a magnet close to the tumor, where these particles act like heat sources, in the 
presence of the applied magnetic field of alternating nature. Hence, the undesirable tissues (cancer's tissues) are destroyed when the temperature reaches $42-45^{\circ} \mathrm{C}$ [6]. Besides, the drug may be placed on the magnetic nanoparticles and is injected near the tumor. The drug is then absorbed by the tumor through a high gradient magnetic field, which is concentrated near the tumor center [26]. In fact, the drug absorption due to high concentration of magnetic particles increases and magnetic force prevents uniform drug distribution in circulatory system. This approach reduces the side effect and allows using high dose of anticancer drug [27].

Very recently, Ebaid and Aly [28] investigated the system of partial differential equations describing the peristaltic flow of a nanofluid with slip effect of the velocity, temperature, and concentration has been analytically solved. The obtained exact solutions have been applied to study effects of the slip parameter, thermophoresis, Brownian motion parameters, and many other parameters on the pressure rise, velocity profiles, temperature distribution, nanoparticle concentration, and pressure gradient. These exact solutions were proved to reduce to the results in the literature at special cases when $\beta_{2}=0$. Moreover, on comparing the present results with those in [22], remarkable differences were noticed for behavior of the included previous physical phenomena.

The main feature of the previous studies on the nanofluid flow under peristaltic action [18-23] is the ignorance of the second slip effect, while this effect has been recently discussed by many authors; see for example [29-33]. These papers showed clearly that the second-order slip flow model is necessary to predict the flow characteristics accurately. Therefore, as an extension of these studies and for the first time, in this paper we aim to investigate effect of the second slip on the peristaltic flow of nanofluids in an asymmetric channel. Hence, we focus here on the problem discussed by Akbar et al. [22] and Ebaid and Aly [28] with slight differences in the boundary conditions.

\section{The Mathematical Model}

Consider peristaltic transport of an incompressible Newtonian nanofluid in an asymmetric channel with flexible walls. The channel asymmetry is generated by propagation of waves on the channel walls traveling with different amplitudes and phases but with the same constant speed $c$. In the Cartesian coordinates system $(\bar{X}, \bar{Y})$ of the fixed frame, the upper and lower walls $\overline{h_{1}}$ and $\overline{h_{2}}$, respectively, are given by (see Figure 1)

$$
\begin{gathered}
\overline{h_{1}}=d_{1}+a_{1} \cos \left(\frac{2 \pi}{\lambda}[\bar{X}-c \bar{t}]\right), \\
\overline{h_{2}}=-d_{2}-b_{1} \cos \left(\frac{2 \pi}{\lambda}[\bar{X}-c \bar{t}]+\phi\right),
\end{gathered}
$$

where $a_{1}$ and $b_{1}$ are amplitude of the waves, $\lambda$ is the wave length, and $d_{1}+d_{2}$ is the width of the channel. The phase difference $\phi$ varies in the range $0 \leq \phi \leq \pi$, where $\phi=0$ and $\phi=\pi$ correspond to symmetric channel with waves out of

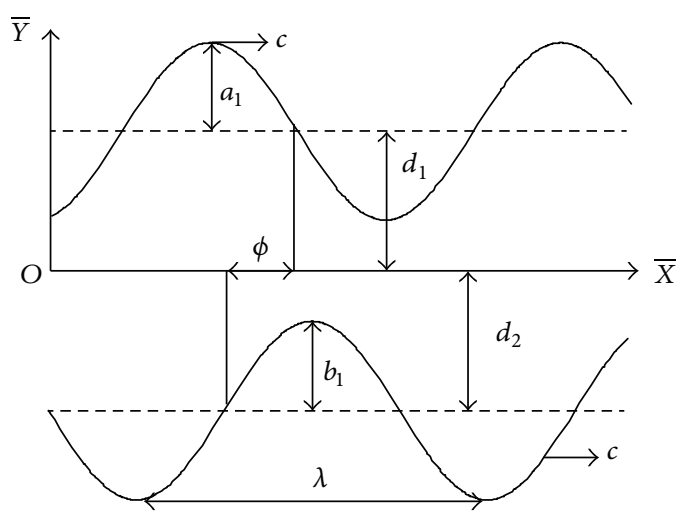

Figure 1: Schematic diagram of a two-dimensional asymmetric channel.

the phase and in the phase, respectively. Further, $a_{1}, b_{1}, d_{1}$, $d_{2}$, and $\phi$ have to satisfy the following condition [34]:

$$
a_{1}^{2}+b_{1}^{2}+2 a_{1} b_{1} \cos \phi \leq\left(d_{1}+d_{2}\right)^{2}
$$

with the following nondimensional phenomena [22]:

$$
a=\frac{a_{1}}{d_{1}}, \quad b=\frac{a_{2}}{d_{1}}, \quad d=\frac{d_{2}}{d_{1}} .
$$

Furthermore, in the moving frame of references $(\bar{x}, \bar{y})$, we have

$$
\bar{x}=\bar{X}-c \bar{t}, \quad \bar{y}=\bar{Y},
$$

which have been used with the following nondimensional phenomena [22]:

$$
\begin{gathered}
x=\frac{2 \pi \bar{x}}{\lambda}, \quad y=\frac{\bar{y}}{d_{1}}, \quad t=\frac{2 \pi \bar{t}}{\lambda}, \\
h_{1}=\frac{\overline{h_{1}}}{d_{1}}, \quad h_{2}=\frac{\overline{h_{2}}}{d_{2}},
\end{gathered}
$$

to obtain the present physical model. On taking into account (i) heat transfer along with nanoparticle phenomena, (ii) long wavelength, and low Reynolds number approximation, recently, Akbar et al. [22] found that the flow is governed by the following system of partial differential equations in nondimensional form:

$$
\begin{gathered}
\frac{\partial^{4} \psi}{\partial y^{4}}+G_{r} \frac{\partial \theta}{\partial y}+B_{r} \frac{\partial \sigma}{\partial y}=0, \\
\frac{d p}{d x}=\frac{\partial}{\partial y}\left[\frac{\partial^{2} \psi}{\partial y^{2}}+G_{r} \theta+B_{r} \sigma\right] \\
\frac{\partial^{2} \theta}{\partial y^{2}}+N_{b} \frac{\partial \theta}{\partial y} \frac{\partial \sigma}{\partial y}+N_{t}\left[\frac{\partial \theta}{\partial y}\right]^{2}=0, \\
\frac{\partial^{2} \sigma}{\partial y^{2}}+\left[\frac{N_{t}}{N_{b}}\right] \frac{\partial^{2} \theta}{\partial y^{2}}=0,
\end{gathered}
$$


where $\psi, \theta, \sigma$, and $p$ are the stream function, temperature distribution, nanoparticle concentration, and pressure gradient, respectively. In addition, $N_{b}, N_{t}, G_{r}$, and $B_{r}$ are the Brownian motion parameter, thermophoresis parameter, local temperature Grashof number, and nanoparticle Grashof number, respectively. The above system of PDEs. (6)-(9) has to be solved subject to the following boundary conditions:

$$
\begin{gathered}
\psi=\frac{F}{2}, \quad \frac{\partial \psi}{\partial y}=-\beta_{1} \frac{\partial^{2} \psi}{\partial y^{2}}-\beta_{2} \frac{\partial^{3} \psi}{\partial y^{3}}-1 \\
\text { at } h_{1}=1+a \cos (x), \\
\psi=-\frac{F}{2}, \quad \frac{\partial \psi}{\partial y}=\beta_{1} \frac{\partial^{2} \psi}{\partial y^{2}}+\beta_{2} \frac{\partial^{3} \psi}{\partial y^{3}}-1 \\
\text { at } h_{2}=-d-b \cos (x+\phi), \\
\theta+\gamma \frac{\partial \theta}{\partial y}=0, \quad \text { at } y=h_{1}, \\
\theta-\gamma \frac{\partial \theta}{\partial y}=1, \quad \text { at } y=h_{2}, \\
\sigma+\gamma_{1} \frac{\partial \sigma}{\partial y}=0, \quad \text { at } y=h_{1}, \\
\sigma-\gamma_{1} \frac{\partial \sigma}{\partial y}=1, \quad \text { at } y=h_{2},
\end{gathered}
$$

where $\beta_{1}, \beta_{2}, \gamma$, and $\gamma_{1}$ represent the first-order slip parameter, second-order slip parameter, thermal slip parameter, and concentration slip parameter, respectively. It should be noted that the full formulation of the above model can be checked in [22], and there is no need to repeat it again here. However, as mentioned in Section 1 in the present work, we focused on obtaining the general closed form solution in the presence of the second-order slip parameter, as a very important one to predict the investigated physical model.

\section{The General Closed Form Solution}

As mentioned by Ebaid and Aly [28], we have the following exact solutions for the temperature distribution and nanoparticle concentration:

$$
\begin{gathered}
\theta(x, y)=f_{4} e^{-N_{b} f_{1} y}+\frac{1}{N_{b}} \frac{f_{3}}{f_{1}}, \\
\sigma(x, y)=-\frac{N_{t}}{N_{b}} f_{4} e^{-N_{b} f_{1} y}+f_{1} y+f_{2}-\frac{N_{t}}{N_{b}^{2}} \frac{f_{3}}{f_{1}},
\end{gathered}
$$

where

$$
\begin{gathered}
f_{2}=\frac{N_{t}}{N_{b}^{2}} \frac{f_{3}}{f_{1}}-\left(\gamma_{1} f_{1}-\frac{1}{N_{b}}\right) N_{t} f_{4} r_{1}^{f_{1}}-\left(\gamma_{1}+h_{1}\right) f_{1}, \\
f_{3}=\frac{-N_{b} f_{1}\left(1-\gamma N_{b} f_{1}\right) r_{1}^{f_{1}}}{\left(1+\gamma N_{b} f_{1}\right) r_{2}^{f_{1}}-\left(1-\gamma N_{b} f_{1}\right) r_{1}^{f_{1}}}, \\
f_{4}=\frac{1}{\left(1+\gamma N_{b} f_{1}\right) r_{2}^{f_{1}}-\left(1-\gamma N_{b} f_{1}\right) r_{1}^{f_{1}}},
\end{gathered}
$$

where

$$
r_{1}=e^{-N_{b} h_{1}}, \quad r_{2}=e^{-N_{b} h_{2}}
$$

$f_{1}$ can be obtained from the following implicit algebraic equation:

$$
\begin{aligned}
\frac{N_{t}}{N_{b}}[ & \left.\frac{\left(\gamma_{1} N_{b} f_{1}-1\right) r_{1}^{f_{1}}+\left(\gamma_{1} N_{b} f_{1}+1\right) r_{2}^{f_{1}}}{\left(\gamma N_{b} f_{1}-1\right) r_{1}^{f_{1}}+\left(\gamma N_{b} f_{1}+1\right) r_{2}^{f_{1}}}\right] \\
& +\left(2 \gamma_{1}+h_{1}-h_{2}\right) f_{1}=-1 .
\end{aligned}
$$

It can be seen from (14) that it is of complex structure to be solved exactly in its general form. However, it has an exact solution for $f_{1}$ when $\gamma=\gamma_{1}$ and given by

$$
f_{1}=\frac{-\left(1+N_{t} / N_{b}\right)}{\left(2 \gamma_{1}+h_{1}-h_{2}\right)} \text {. }
$$

Regarding the stream function, it can be solved to give

$$
\psi=f_{8}+f_{7} y+\frac{1}{2} f_{6} y^{2}+\frac{1}{6} f_{5} y^{3}+g(y),
$$

where

$$
g(y)=\frac{1}{6} \Omega_{1} y^{3}-\frac{1}{24} B_{r} f_{1} y^{4}-\frac{\Omega_{2}}{\left(N_{b} f_{1}\right)^{3}} e^{-N_{b} f_{1} y}
$$

Applying the boundary conditions on the $\psi$-equation, we obtain the following system:

$$
\begin{gathered}
f_{8}+f_{7} h_{1}+\frac{1}{2} f_{6} h_{1}^{2}+\frac{1}{6} f_{5} h_{1}^{3}=R_{1}, \\
f_{8}+f_{7} h_{2}+\frac{1}{2} f_{6} h_{2}^{2}+\frac{1}{6} f_{5} h_{2}^{3}=R_{2}, \\
f_{7}+\left(\beta_{1}+h_{1}\right) f_{6}+\left(\frac{1}{2} h_{1}^{2}+\beta_{1} h_{1}\right) f_{5}=S_{1}, \\
f_{7}+\left(h_{2}-\beta_{1}\right) f_{6}+\left(\frac{1}{2} h_{2}^{2}-\beta_{1} h_{2}-\beta_{2}\right) f_{5}=S_{2},
\end{gathered}
$$

where

$$
\begin{gathered}
R_{1}=\frac{F}{2}-g\left(h_{1}\right), \\
R_{2}=-\frac{F}{2}-g\left(h_{2}\right), \\
S_{1}=-1-g^{\prime}\left(h_{1}\right)-\beta_{1} g^{\prime \prime}\left(h_{1}\right)-\beta_{2} g^{\prime \prime \prime}\left(h_{1}\right), \\
S_{2}=-1-g^{\prime}\left(h_{2}\right)+\beta_{1} g^{\prime \prime}\left(h_{2}\right)+\beta_{2} g^{\prime \prime \prime}\left(h_{2}\right) .
\end{gathered}
$$


On solving the system (18a)-(18d), we get

$$
\begin{aligned}
& f_{5}=\frac{6\left(-2 R_{1}+2 R_{2}+\left(h_{1}-h_{2}\right)\left(S_{1}+S_{2}\right)\right)}{\left(h_{1}-h_{2}\right)^{2}\left(6 \beta_{1}+h_{1}-h_{2}\right)}, \\
& f_{6}=\left(2 \left(-h_{1}^{3}\left(S_{1}+2 S_{2}\right)+6 h_{1}^{2}\left(R_{1}-R_{2}+S_{2}\left(h_{2}-2 \beta_{1}\right)\right)\right.\right. \\
& +2 h_{2}\left(-h_{2}\left(3 R_{1}-3 R_{2}+h_{2}\left(2 S_{1}+S_{2}\right)\right)\right. \\
& \left.+6\left(R_{1}-R_{2}+h_{2} S_{1}\right) \beta_{1}\right) \\
& +6 h_{1}\left(h_{2}^{2} S_{1}+2\left(R_{1}-R_{2}+h_{2}\left(-S_{1}+S_{2}\right)\right) \beta_{1}\right) \\
& \left.\left.+12\left(2 R_{1}-2 R_{2}-\left(h_{1}-h_{2}\right)\left(S_{1}-S_{2}\right)\right) \beta_{2}\right)\right) \\
& \times\left(\left(\left(h_{1}-h_{2}\right)^{2}\left(h_{1}-h_{2}+2 \beta_{1}\right)\left(h_{1}-h_{2}+6 \beta_{1}\right)\right)\right)^{-1}, \\
& f_{7}=\left(h_{1}^{4} S_{2}+2 h_{1}^{3}\left(h_{2} S_{1}-\left(S_{1}-2 S_{2}\right) \beta_{1}\right)\right. \\
& +2 h_{1}\left(h_{2}^{3} S_{2}+12 h_{2}\left(-R_{1}+R_{2}\right) \beta_{1}\right. \\
& +3 h_{2}^{2}\left(R_{1}-R_{2}-S_{2} \beta_{1}\right) \\
& \left.+6\left(R_{1}-R_{2}\right)\left(\beta_{1}^{2}-\beta_{2}\right)\right) \\
& +h_{1}^{2}\left(-3 h_{2}\left(2 R_{1}-2 R_{2}+h_{2}\left(S_{1}+S_{2}\right)\right)\right. \\
& \left.+6\left(R_{1}-R_{2}+h_{2} S_{1}\right) \beta_{1}+6\left(S_{1}+S_{2}\right) \beta_{2}\right) \\
& +h_{2}\left(h_{2}^{3} S_{1}+2 h_{2}^{2}\left(-2 S_{1}+S_{2}\right) \beta_{1}-12\left(R_{1}-R_{2}\right)\right. \\
& \times\left(\beta_{1}^{2}+\beta_{2}\right) \\
& \left.\left.+6 h_{2}\left(\left(R_{1}-R_{2}\right) \beta_{1}-\left(S_{1}+S_{2}\right) \beta_{2}\right)\right)\right) \\
& \times\left(\left(h_{1}-h_{2}\right)^{2}\left(h_{1}-h_{2}+2 \beta_{1}\right)\left(h_{1}-h_{2}+6 \beta_{1}\right)\right)^{-1}, \\
& f_{8}=\left(h_{1}^{4}\left(R_{2}-h_{2} S_{2}\right)-h_{1}^{3}\left(4 R_{2}+h_{2}\left(S_{1}-S_{2}\right)\right)\left(h_{2}-2 \beta_{1}\right)\right. \\
& +h_{2}^{2} R_{1}\left(h_{2}-6 \beta_{1}\right)\left(h_{2}-2 \beta_{1}\right) \\
& +h_{1}^{2}\left(h_{2}^{3}\left(2 S_{1}-S_{2}\right)+12 R_{2} \beta_{1}^{2}\right. \\
& +3 h_{2}^{2}\left(R_{1}+R_{2}+2\left(-S_{1}+S_{2}\right) \beta_{1}\right) \\
& \left.+6 h_{2}\left(-\left(R_{1}+3 R_{2}\right) \beta_{1}-\left(S_{1}+S_{2}\right) \beta_{2}\right)\right) \\
& +h_{1} h_{2}\left(-h_{2}^{3} S_{1}-12\left(R_{1}+R_{2}\right) \beta_{1}^{2}\right. \\
& -2 h_{2}^{2}\left(2 R_{1}+\left(-2 S_{1}+S_{2}\right) \beta_{1}\right) \\
& +12\left(R_{1}-R_{2}\right) \beta_{2} \\
& \left.\left.+6 h_{2}\left(\left(3 R_{1}+R_{2}\right) \beta_{1}+\left(S_{1}+S_{2}\right) \beta_{2}\right)\right)\right) \\
& \times\left(\left(h_{1}-h_{2}\right)^{2}\left(h_{1}-h_{2}+2 \beta_{1}\right)\left(h_{1}-h_{2}+6 \beta_{1}\right)\right)^{-1} \text {. }
\end{aligned}
$$

To get the pressure gradient $d p / d x$, we obtain from (7) that

$$
\frac{d p}{d x}=\Omega_{3}-B_{r} f_{1} y+\left(1+N_{b} f_{1}\right) \Omega_{2} e^{-N_{b} f_{1} y},
$$

where

$$
\begin{gathered}
\Omega_{3}=\Omega_{1}+f_{5}+B_{r} f_{1}, \quad \Omega_{2}=\left(\frac{B_{r} N_{t}}{N_{b}}-G_{r}\right) f_{4}, \\
\Omega_{1}=\left(\frac{B_{r} N_{t}}{N_{b}}-G_{r}\right) \frac{1}{N_{b}} \frac{f_{3}}{f_{1}}-B_{r} f_{2} .
\end{gathered}
$$

The pressure rise $\Delta p$ in terms of the flow rate $Q$ is given as follows:

$$
\Delta p=\int_{0}^{1}\left(\frac{d p}{d x}\right) d x
$$

Therefore, the exact expression for the pressure rise at the center of the channel is given as

$$
\begin{aligned}
\Delta p=\int_{0}^{1}\left[\frac{6\left[2 g\left(h_{1}\right)-2 g\left(h_{2}\right)+\left(h_{1}-h_{2}\right)\left(S_{1}+S_{2}\right)\right]}{\left(h_{1}-h_{2}\right)^{2}\left(6 \beta_{1}+h_{1}-h_{2}\right)}\right. \\
\quad+\Omega_{1}+B_{r} f_{1}\left(1-\frac{h_{1}+h_{2}}{2}\right) \\
\left.\quad+\left(1+N_{b} f_{1}\right) \Omega_{2} e^{\left.-N_{b} f_{1}\left(h_{1}+h_{2}\right) / 2\right)}\right] d x \\
-12(Q-1-d) \int_{0}^{1} \frac{1}{\left(h_{1}-h_{2}\right)^{2}\left(6 \beta_{1}+h_{1}-h_{2}\right)} d x .
\end{aligned}
$$

\section{Results and Discussion}

This section is devoted for investing the exact solutions which have been obtained in the previous section for the temperature distribution, nanoparticle concentration, velocity, pressure gradient, and pressure rise. These solutions are expected to provide us with the correct physical effect of the second slip for the five investigated physical phenomena. It is observed from (11) that there is no effect for the second slip parameter on the solutions of the temperature distribution and nanoparticle concentration. Although (11) have been used by Ebaid and Aly [28] to discuss the effect of many parameters on the temperature distribution and nanoparticle concentration for comparing with the results in [22], many other parameters were ignored. These important parameters are investigated in the next subsections.

4.1. The Temperature Distribution $\theta$. Very recently in [28], Ebaid and Aly discussed the effect of $N_{t}, \gamma$, and $\gamma_{1}$ on the temperature distribution. Here, we aim to discuss the effect of many other parameters on this phenomenon. In Figures 2(a) and 2(b), effect of the amplitude $a$ on the temperature distribution is depicted at two different values for the Browning motion parameter and fixed values for the 


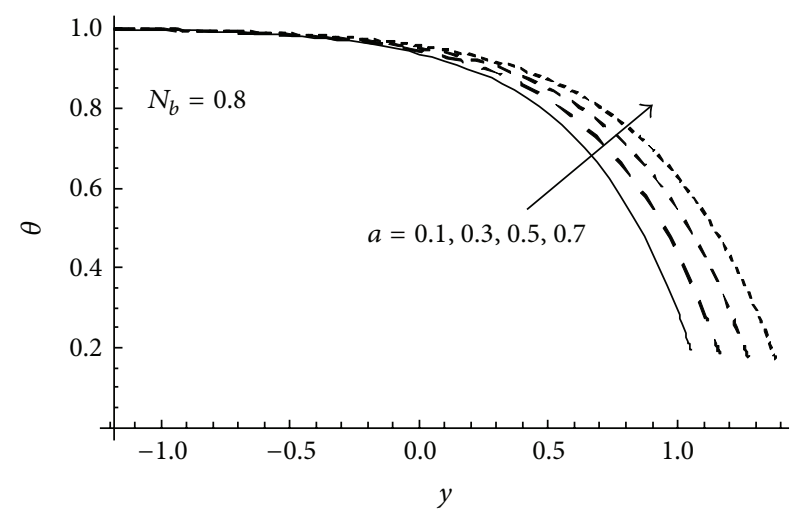

(a)

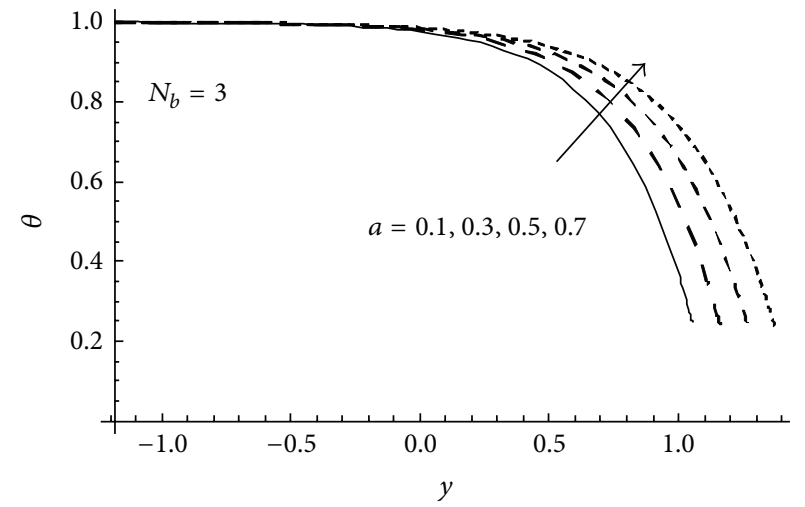

(b)

FIGURE 2: Variation of temperature profile at different values of $a$ when $N_{t}=5, d=1, b=0.5, x=1, \phi=0.2, \gamma=0.1$, and $\gamma_{1}=0.1$ for (a) $N_{b}=0.8$ and (b) $N_{b}=3$.

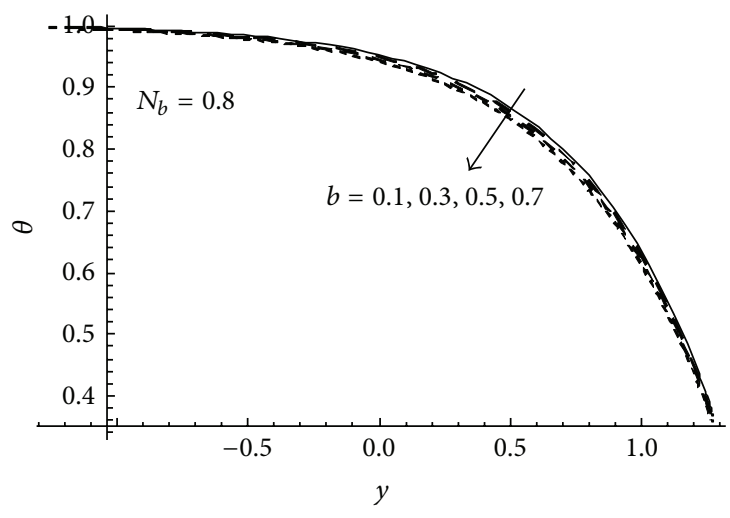

(a)

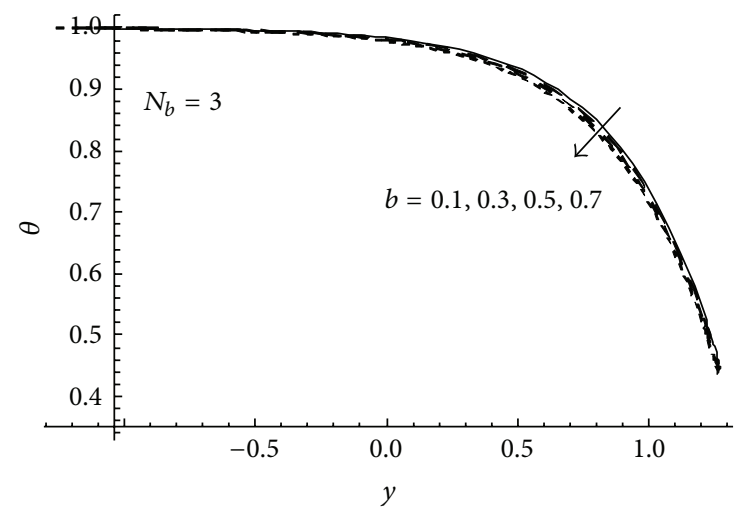

(b)

Figure 3: Variation of temperature profile at different values of $b$ when $N_{t}=5, d=1, a=0.5, x=1, \phi=0.2, \gamma=0.3$, and $\gamma_{1}=0.3$ for (a) $N_{b}=0.8$ and (b) $N_{b}=3$.

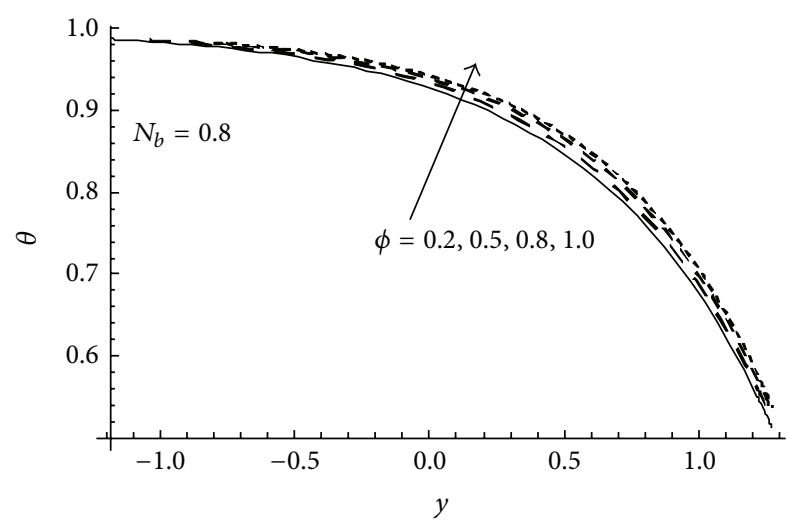

(a)

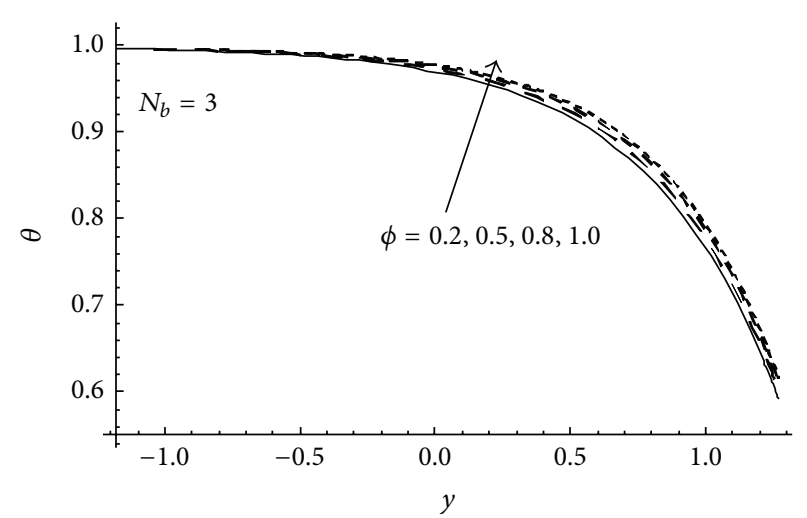

(b)

Figure 4: Variation of temperature profile at different values of $\phi$ when $N_{t}=5, d=1, a=0.5, x=1, b=0.5, \gamma=0.7$, and $\gamma_{1}=0.7$ for (a) $N_{b}=0.8$ and (b) $N_{b}=3$. 


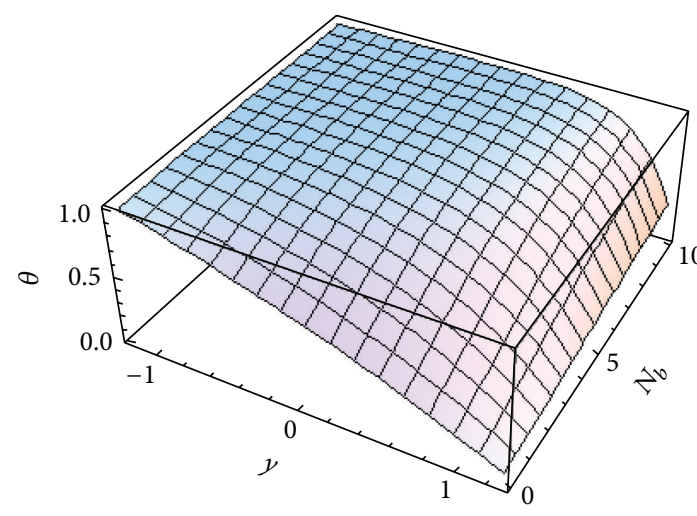

(a)

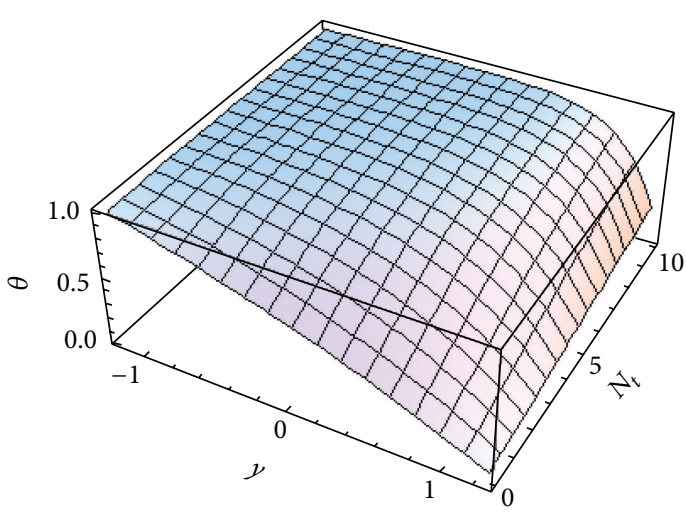

(b)

FIGURE 5: Variation of temperature profile at different values of (a) $N_{b}$ for $N_{t}=0.8$ and (b) $N_{t}$ for $N_{b}=0.8$; both cases when $d=1, a=0.5$, $b=0.5, \phi=0.2, x=1, \gamma=0.1$, and $\gamma_{1}=0.1$.

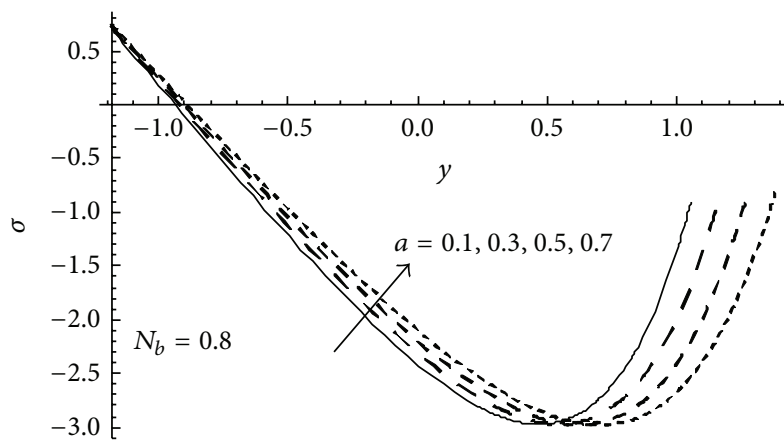

(a)

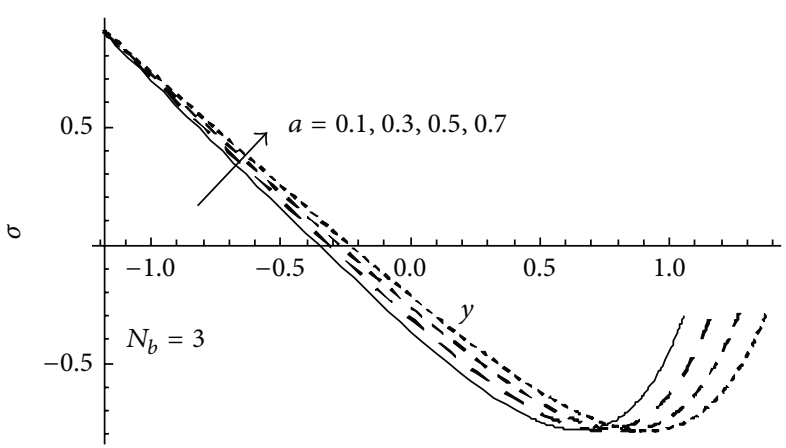

(b)

FIGURE 6: Variation of nanoparticle concentration at different values of $a$ when $N_{t}=5, d=1, b=0.5, x=1, \phi=0.2, \gamma=0.1$, and $\gamma_{1}=0.1$ for (a) $N_{b}=0.8$ and (b) $N_{b}=3$.

other investigated parameters. From these figures, it can be concluded that there are no remarkable influences for the variation of $a$ on $\theta$ in the first part of the channel, while an increase in $\theta$ occurs in the upper part with increasing $a$. The variation of the amplitude $b$ on the temperature distribution is presented in Figures 3(a) and 3(b). These figures show that very small decrease in $\theta$ occurs as $b$ increases. This means that while the amplitude $a$ has remarkable effect on $\theta$, the influence of the amplitude $b$ on $\theta$ may be ignored. In Figures 4(a) and 4(b), the variation of the phase difference $\phi$ on $\theta$ is introduced. Although the increase in $\phi$ between the waves of the channel increases the temperature distribution, this increase is very small. Instead of studying the variation of $\theta$ at separate values of Brownian motion parameter $N_{b}$, we present in Figure 5(a) the variation of $\theta$ against a continuous range for the values of $N_{b}$. This figure indicates a clear description for the variation of Brownian motion parameter $N_{b}$ on $\theta$. In addition, Figure 5(b) shows other descriptions for the variation of the thermophoresis parameter $N_{t}$ on the temperature distribution. It can be concluded from the last two figures that $\theta$ always decreases with the increase in $N_{b}$ and $N_{t}$.
4.2. The Nanoparticle Concentration $\sigma$. The nanoparticles concentration $\sigma$ is depicted in Figures 6(a) and 6(b). It is observed from these figures that $\sigma$ reaches its highest value at the lower wall of the channel and then it decreases in a certain domain across the channel. A converse of this behavior occurs after that domain. It is also detected that at a higher value of Brownian motion parameter $N_{b}$ the domain in which $\sigma$ decreases becomes wider. In addition, the wide of such domain increases with increasing the amplitude of the upper wave. The variation of the lower wave amplitude on $\sigma$ is presented in Figures 7(a) and 7(b). For all values of $b$, it is noticed that $\sigma$ decreases until it reaches a certain point. After that point the curves become identical whatever the value of $b$ becomes. This refers to that there is no effect for $b$ on $\sigma$ after this point.

In Figures $8(\mathrm{a})$ and $8(\mathrm{~b})$, the variation of the phase difference $\phi$ on $\sigma$ is displayed. Although the increase in the phase difference between the waves of the channel increases the nanoparticles concentration, this occurs in certain domain of the channel. However, after that domain the curves are identical whatever the value of the phase difference. Moreover, instead of studying the variation of $\sigma$ 


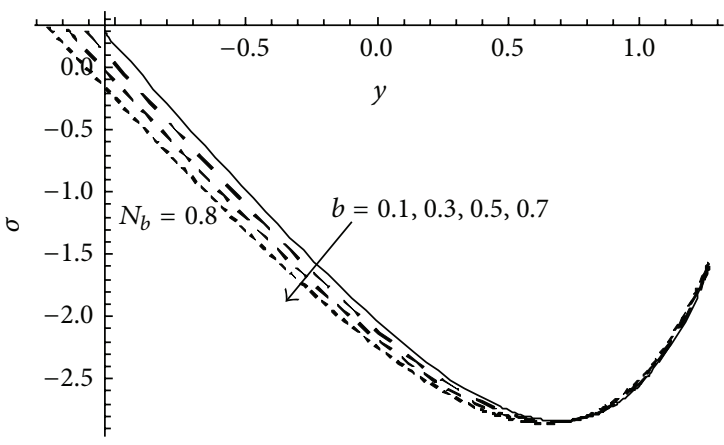

(a)

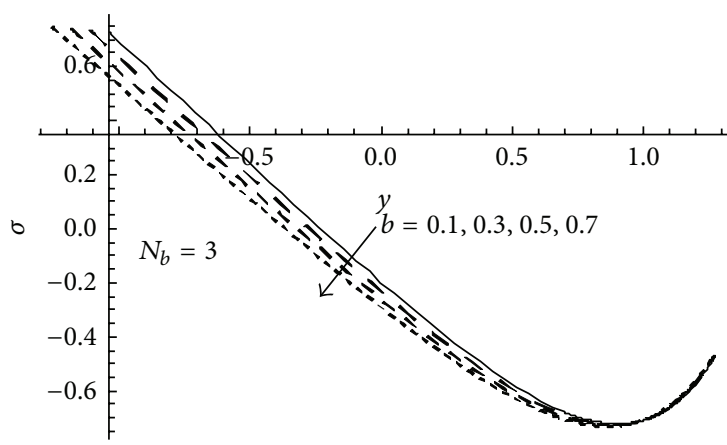

(b)

FIGURE 7: Variation of nanoparticle concentration at different values of $b$ when $N_{t}=5, d=1, a=0.5, x=1, \phi=0.2, \gamma=0.3$, and $\gamma_{1}=0.3$ for (a) $N_{b}=0.8$ and (b) $N_{b}=3$.

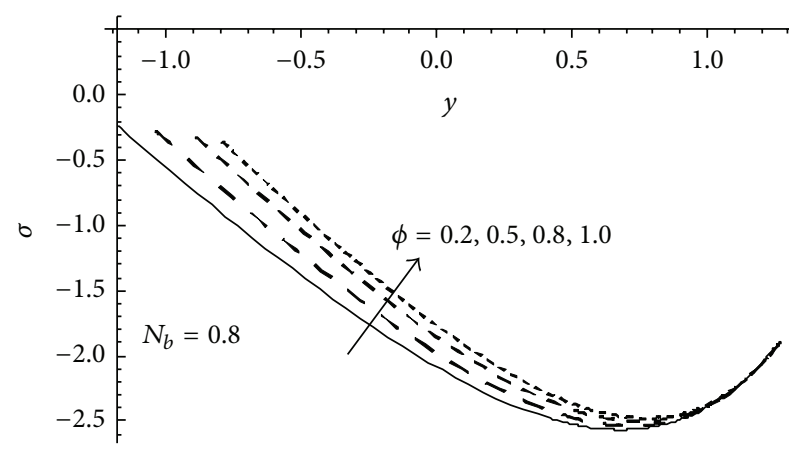

(a)

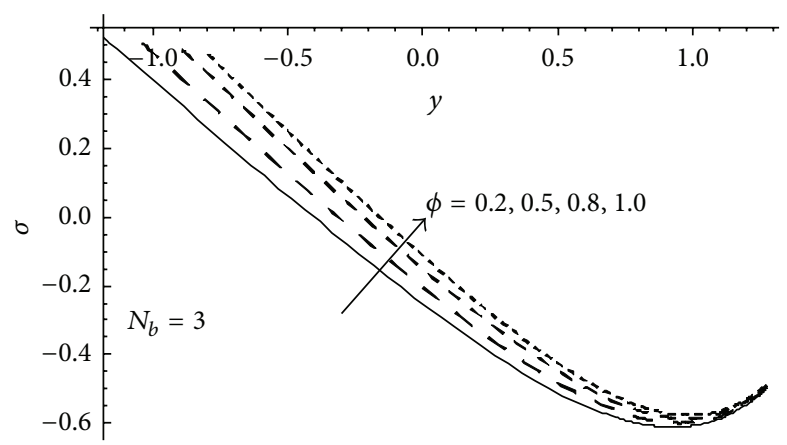

(b)

FIGURE 8: Variation of nanoparticle concentration at different values of $\phi$ when $N_{t}=5, d=1, a=0.5, x=1, b=0.5, \gamma=0.7$, and $\gamma_{1}=0.7$ for (a) $N_{b}=0.8$ and (b) $N_{b}=3$.

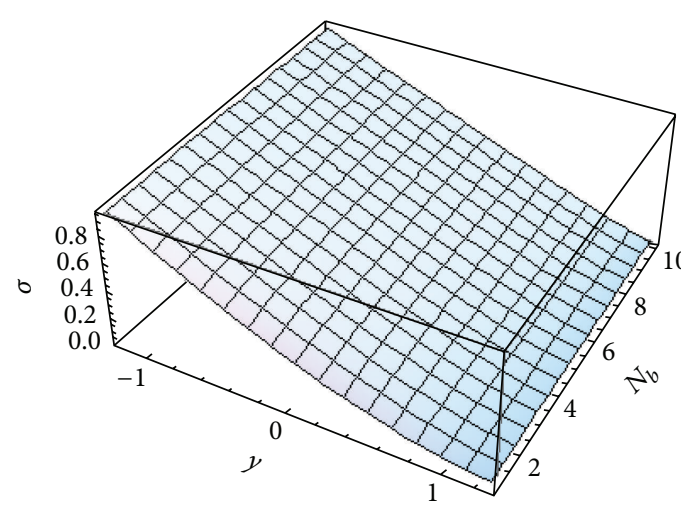

(a)

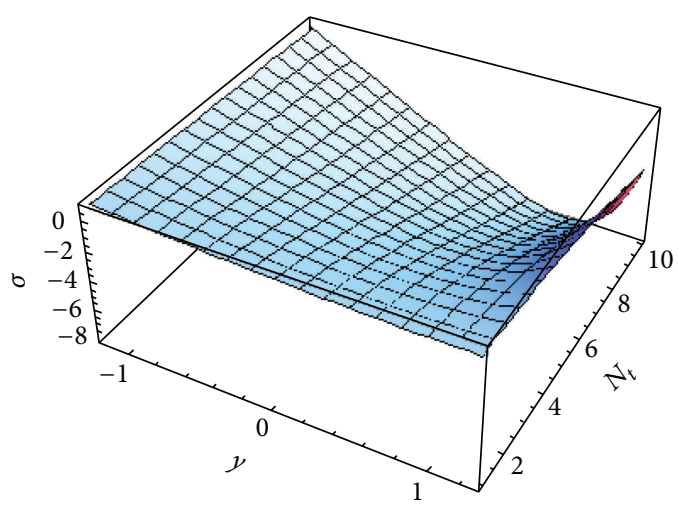

(b)

Figure 9: Variation of nanoparticle concentration at different values of (a) $N_{b}$ for $N_{t}=0.8$ and $q=0.5$ and (b) $N_{t}$ for $N_{b}=0.8$ and $b=0.5$; both cases when $d=1, a=0.5, \phi=0.2, x=1, \gamma=0.1$, and $\gamma_{1}=0.1$.

at separate values of Brownian motion parameter $N_{b}$, the variation of $\sigma$ against a continuous range for the values of $N_{b}$ is displayed in Figure 9(a). In addition, Figure 9(b) shows other descriptions for the variation of the thermophoresis parameter $N_{t}$ on the nanoparticle concentration. It can be concluded from Figure 9(a) that $\sigma$ always decreases when $N_{b}$ is in the range from 1 to 10 at fixed values for the other parameters. However, the situation is different for the variation of $\sigma$ against $y$ at certain values of $N_{t}$, where it is noticed that when $1<N_{t}<4$ then $\sigma$ decreases within the 


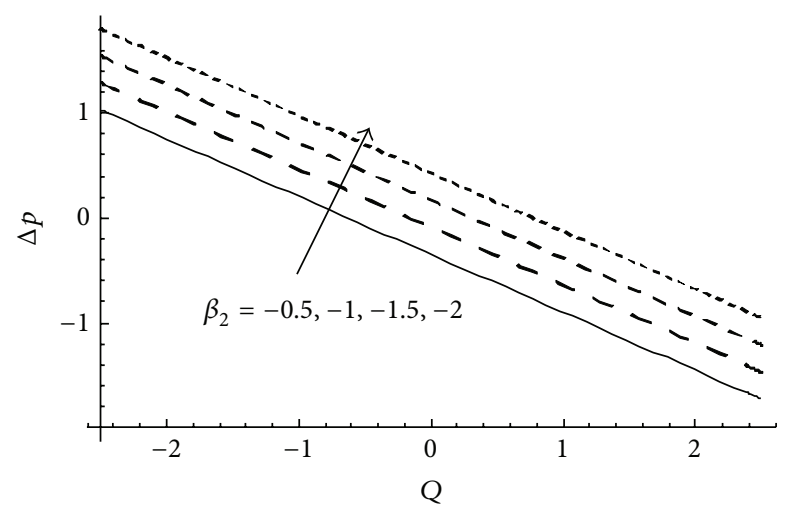

(a)

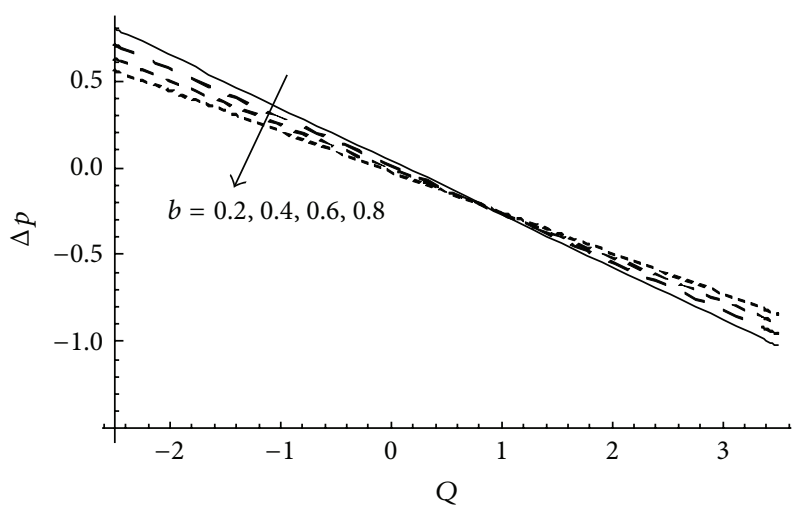

(c)

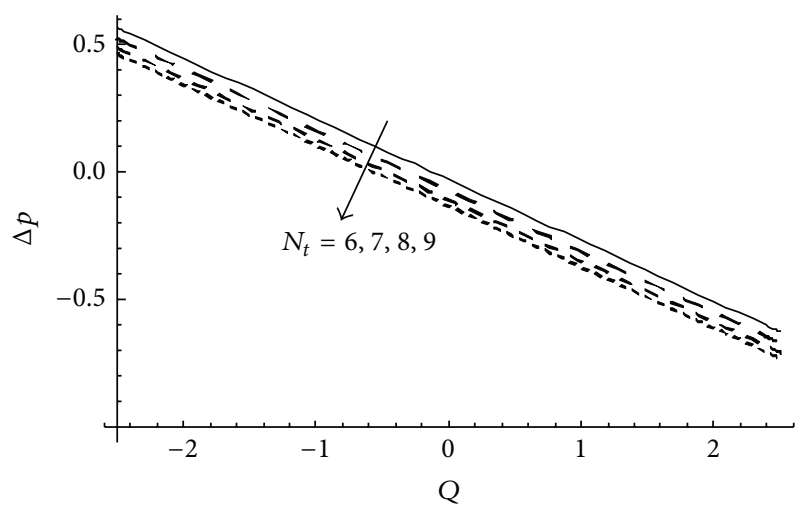

(b)

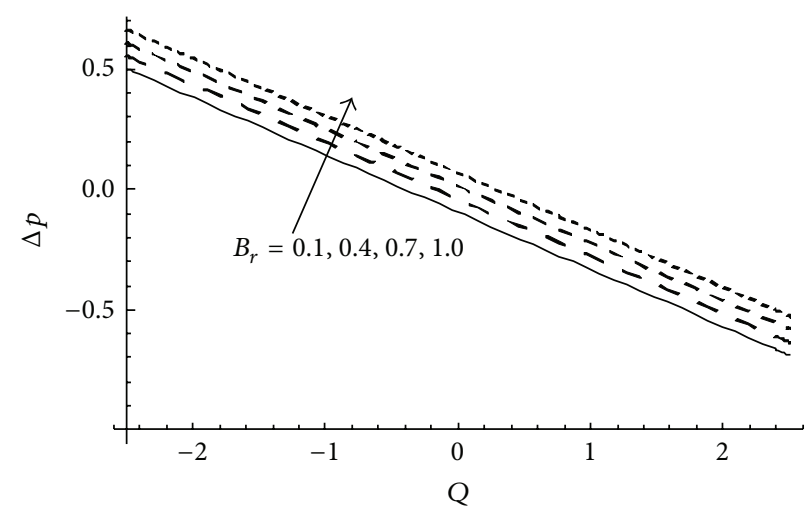

(d)

FIGURE 10: Variation of the pressure rise versus flow rate at $a=0.3, d=1$, and $\gamma=\gamma_{1}=0.5$ for (a) $b=0.5, N_{b}=0.8, N_{t}=0.5, B_{r}=0.5$, $G_{r}=0.2, \phi=0.2$, and $\beta_{1}=0.1$; (b) $b=0.5, N_{b}=0.5, B_{r}=0.5, G_{r}=0.5, \phi=0.2, \beta_{1}=0.8$, and $\beta_{2}=-2$; (c) $N_{b}=0.8, N_{t}=0.5, B_{r}=0.5$, $G_{r}=0.2, \phi=0.5, \beta_{1}=0.8$, and $\beta_{2}=-2$; and (d) $b=0.5, N_{b}=0.8, N_{t}=0.5, G_{r}=0.2, \phi=0.2, \beta_{1}=0.8$, and $\beta_{2}=-2$.

channel. In addition, at values of $N_{t}$ higher than 4 , we observe that $\sigma$ decreases in certain domain of the channel and changes this behavior in the rest of channel.

4.3. The Pressure Rise $\Delta p$. The exact expression for the pressure rise is given by (24) which is valid for any set of the physical parameters. On using this equation, we observe that the pressure rise is always a decreasing function in terms of the flow rate. This notice is clarified in Figures 10(a)-10(d). It is also observed from Figure 10(a) that the pressure rise increases with increasing the negativity of the second slip parameter $\beta_{2}$ in the whole range of the flow rate $Q$. The effect of the thermophoresis parameter $N_{t}$ on the pressure rise is depicted in Figure 10(b). It can be seen from this figure that the pressure rise decreases with the increase in the values of $N_{t}$ in the whole region of $Q$.

Figure 10(c) shows that the pressure rise decreases with decreasing the amplitude $b$ when $Q$ lies in the region $-2.5<$ $Q<1$, while a converse behavior occurs for $Q>1$. Figure $10(\mathrm{~d})$ reveals that the pressure rise increases with increasing the local nanoparticle Grashof number $B_{r}$. This behavior differs from the results obtained by Akbar et al. [22] in the absence of the second slip effect. Therefore, the present results reveal that the existence of this parameter has a significant effect on the pressure rise and leads to different behavior when compared with its ignorance.

4.4. The Velocity $u(y)$. Regarding the axial velocity, it was plotted in Figures 11(a)-11(d) at several values of the present physical parameters. Variations of the second slip parameter $\beta_{2}$, Brownian motion parameter $N_{b}$, flow rate $Q$, and local temperature Grashof number $G_{r}$ on the velocity profile have been displayed in Figures 11(a)-11(d), respectively. It is found from Figure 11(a) that the velocity increases near the lower wall with increasing $\beta_{2}$, while a converse behavior occurs near the upper wall; that is, the velocity decreases with increasing $\beta_{2}$. Unlike the study made by Akbar et al. [22], the effect of Brownian motion parameter $N_{b}$ leads to that there are three different regions in which alternating behavior for the velocity occurs. As shown in Figure 11(b), in a central region of the channel, that is, $-0.6<y<0.8$, the velocity increases with the increase in $N_{b}$, while in the other two regions of the channel, that is, $h_{2}<y<-0.6$ and $0.8<y<h_{1}$, a different situation is detected. At the same three regions just mentioned, it can be seen from Figure 11(d) that effect of the local temperature Grashof number $G_{r}$ on the velocity profiles happens in a converse manner regarding Brownian motion parameter $N_{b}$. However, it is clear from Figure 11(c) 


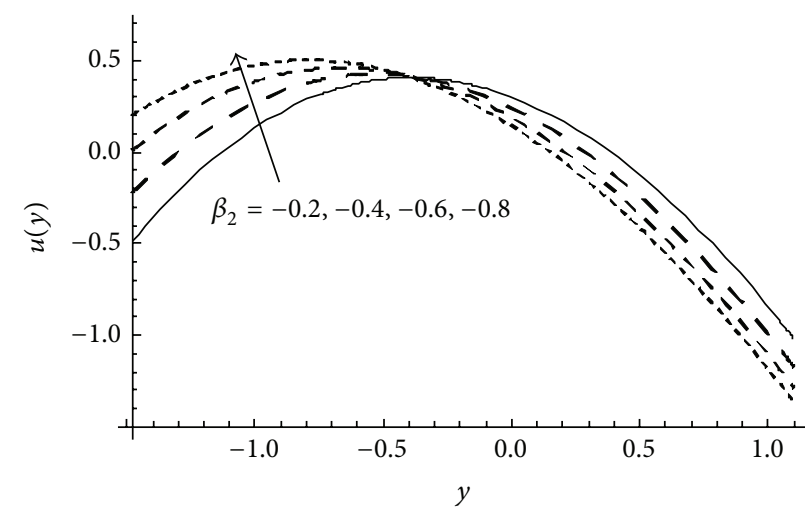

(a)

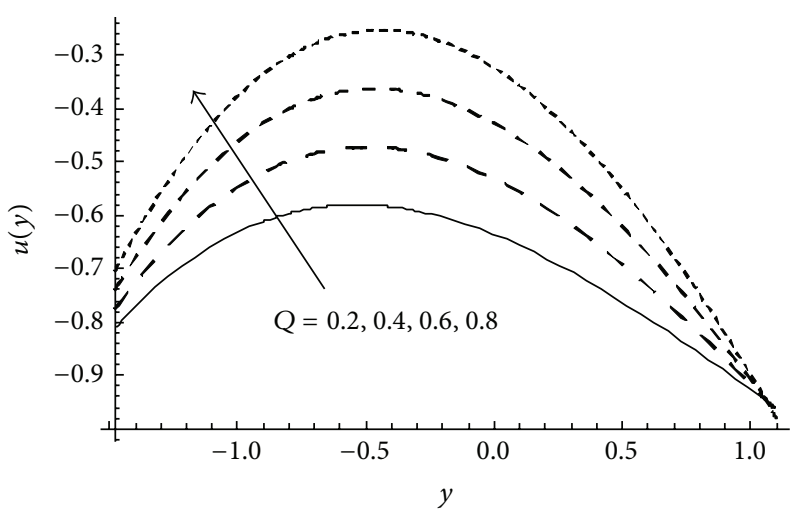

(c)

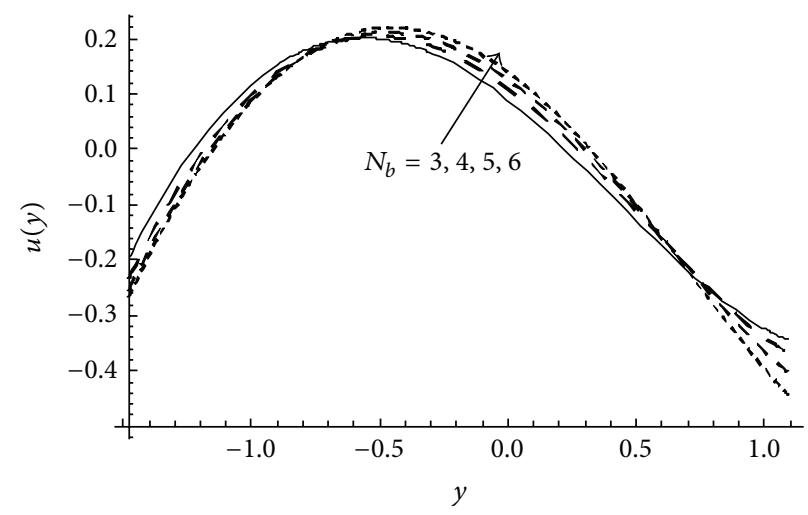

(b)

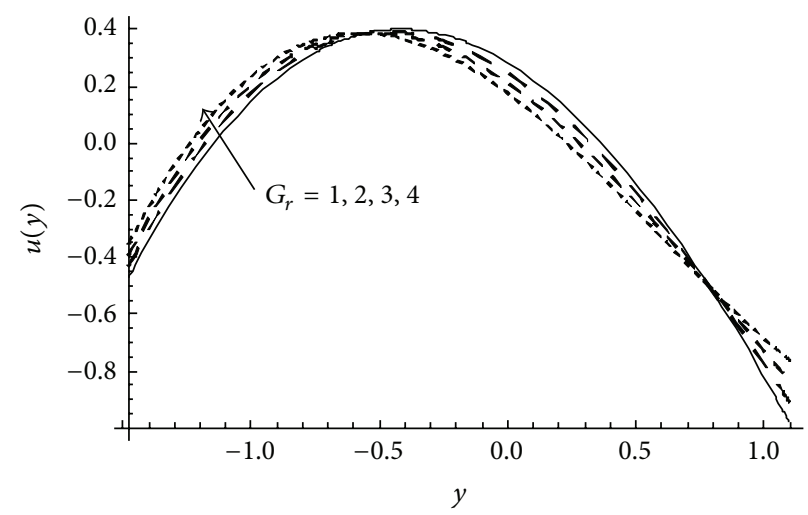

(d)

FIGURE 11: Variation of the velocity profile at $a=0.1, b=0.5, d=1, B_{r}=0.5, x=0.1, \phi=0.2, \gamma=\gamma_{1}=0.5$, and $\beta_{1}=0.1$ for (a) $N_{t}=0.5$, $N_{b}=0.8, G_{r}=0.5, Q=2$, and $\beta_{2}=-0.2,-0.4,-0.6,-0.8$; (b) $N_{t}=0.7, G_{r}=3, Q=2, \beta_{2}=-0.6$, and $N_{b}=3,4,5,6$; (c) $N_{t}=0.5, N_{b}=0.8$, $G_{r}=0.5, \beta_{2}=-0.2$, and $Q=0.2,0.4,0.6,0.8$; and (d) $N_{t}=0.5, N_{b}=0.8, Q=2, \beta_{2}=-0.2$, and $G_{r}=1,2,3,4$.

that an increase in the flow rate leads to an increase in the velocity and this is similar to the variation of the velocity profile against flow rate as in Akbar et al. [22].

4.5. The Pressure Gradient $d p / d x$. Figures $12(\mathrm{a})-12(\mathrm{~d})$ indicate the pressure gradient for different values of $\beta_{2}, N_{b}$, $G_{r}$, and $a$, respectively. From these figures, it is shown that magnitude of the pressure gradient increases in view of an increase in $\beta_{2}, G_{r}$, and $a$, and it decreases with an increase in $N_{b}$. In addition, it is observed that our results on the effect of $\beta_{2}, N_{b}$, and $G_{r}$ on the pressure gradient are completely different comparing with those obtained in [22] for the effect of the first slip parameter only. This also may refer to the importance of including the second slip parameter $\beta_{2}$ in such problems of peristaltic flow. However, the effect of the amplitude $a$ on the pressure gradient remains the same even in the presence of $\beta_{2}$, where Figure $12(\mathrm{~d})$ shows that an increase in the amplitude $a$ leads to an increase in the pressure gradient. Besides, one of the main features of these graphs is that the maximum pressure gradient occurs at the same point when $x=0.5$. In the absence of $\beta_{2}$, this value was shifted slightly to the left; that is, it occurred at $x=0.45$ as in [22]. The final notice here is that the present numerical discussion is based on the obtained exact analytical solutions for the current physical model, unlike the approximate series solutions obtained in [22] whose disadvantages have been proved by Ebaid and Aly [28].

\section{Conclusion}

The effect of the second slip condition on the peristaltic transport of a nanofluid in an asymmetric channel was investigated for the first time in the present paper. The system describing the problem was exactly solved via a simple, but very effective, analytical procedure. Based on the obtained exact solutions, some important results were introduced through graphs to indicate effect of the second slip on the velocity profiles, pressure rise, and pressure gradient. Moreover, the variation of many physical parameters such as amplitudes of the lower and upper waves, phase difference on the temperature distribution, and nanoparticle concentration were also discussed for a new set of the presented parameters rather than those in the literature. It was found that the temperature distribution $\theta$ always decreases with the increase in $N_{b}$ and $N_{t}$. In addition, the amplitude $a$ has remarkable effect on $\theta$, while the influence of the amplitude $b$ on $\theta$ may be ignored. For all values of $b$, nanoparticles concentration $\sigma$ decreases until it reaches a certain point; however, there 


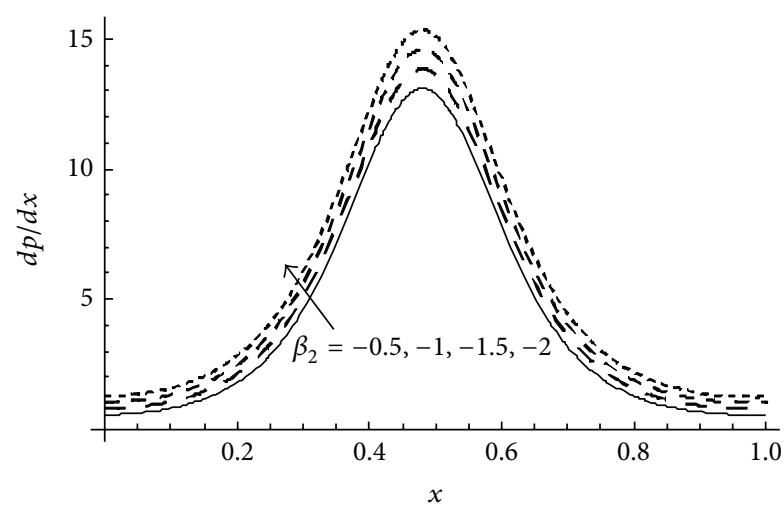

(a)

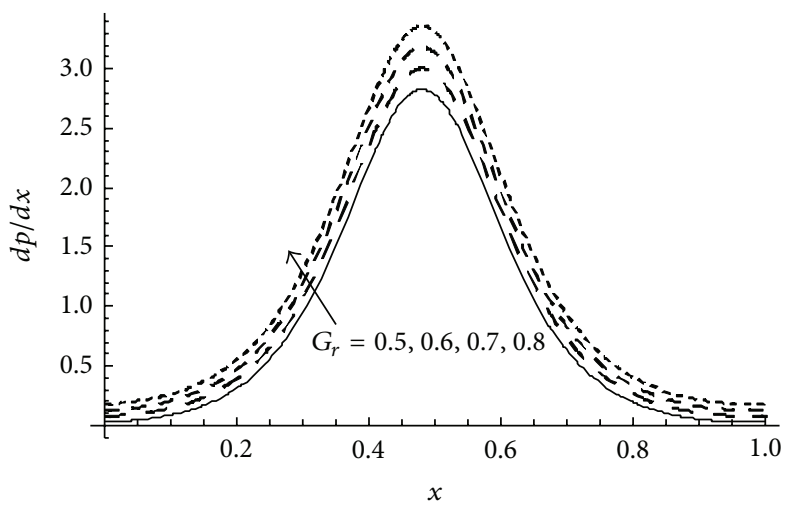

(c)

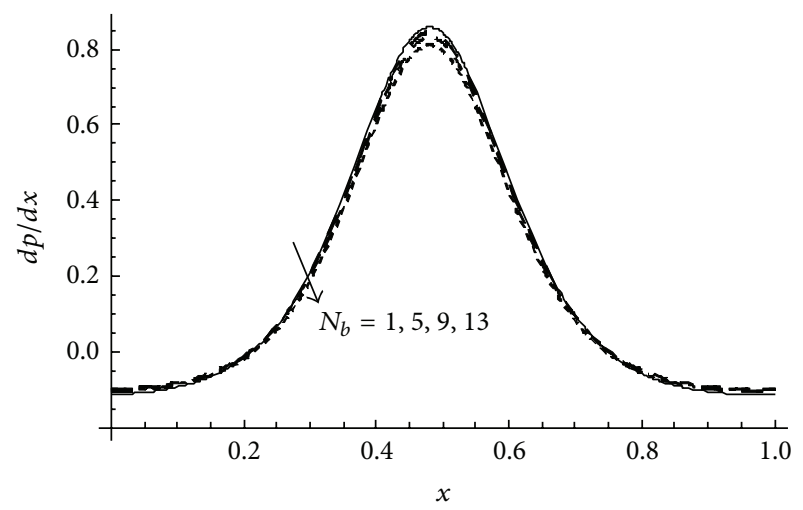

(b)

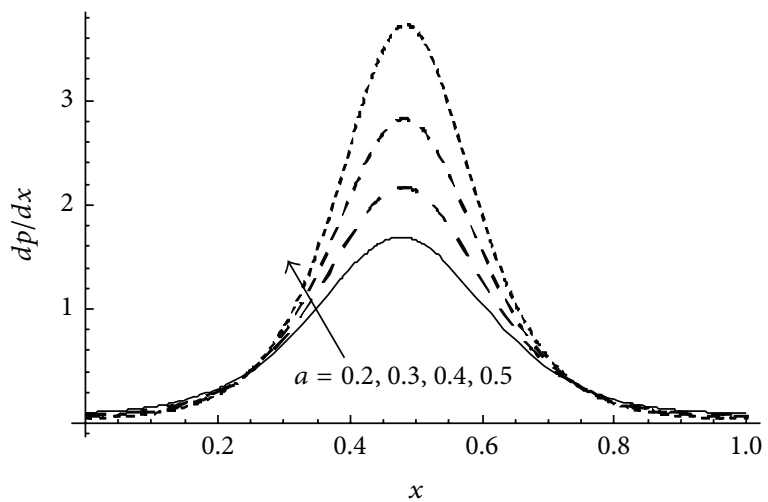

(d)

Figure 12: Variation of the pressure gradient at $b=0.5, d=1, N_{t}=0.5, B_{r}=0.5, \phi=0.2, Q=0.5$, and $\gamma=\gamma_{1}=0.5$ for (a) $\beta_{1}=0.1$, $N_{b}=0.8, G_{r}=0.5, a=0.3$, and $\beta_{2}=-0.5,-1,-1.5,-2$; (b) $\beta_{1}=0.8, \beta_{2}=-2, G_{r}=0.5, a=0.3$, and $N_{b}=1,5,9,13$; (c) $\beta_{1}=0.8, \beta_{2}=-2$, $N_{b}=0.8, a=0.3$, and $G_{r}=0.5,0.6,0.7,0.8$; and (d) $\beta_{1}=0.3, \beta_{2}=-2, N_{b}=0.8, G_{r}=0.5$, and $a=0.2,0.3,0.4,0.5$.

is no effect for $b$ after this point. In a certain domain of the channel, the phase difference between the waves of the channel increases $\sigma$; however, the curves are identical whatever value of the phase difference after that. Finally, it should be mentioned that the second slip condition had a significant effect on the various phenomena involved in the current model. Accordingly, the present suggested technique may be useful for obtaining exact solutions for many other similar problems. In addition, such type of the second slip should be included for modelling the peristaltic flow.

\section{Conflict of Interests}

The authors declare that there is no conflict of interests regarding the publication of this paper.

\section{Acknowledgments}

This paper was funded by the Deanship of Scientific Research (DSR), King Abdulaziz University, Jeddah, under Grant no. (130-023-D1434). The authors, therefore, acknowledge DSR technical and financial support.

\section{References}

[1] T. W. Latham, Fluid motion in a peristaltic pump, [M.Sc. thesis], MIT, Cambridge, Mass, USA, 1966.

[2] A. Ramachandra Rao and S. Usha, "Peristaltic transport of two immiscible viscous fluids in a circular tube," Journal of Fluid Mechanics, vol. 298, pp. 271-285, 1995.

[3] J. C. Misra and S. K. Pandey, "Peristaltic transport in a tapered tube," Mathematical and Computer Modelling, vol. 22, no. 8, pp. 137-151, 1995.

[4] K. S. Mekheimer, "Peristaltic transport of a couple stress fluid in a uniform and non-uniform channels," Biorheology, vol. 39, no. 6, pp. 755-765, 2002.

[5] K. Vajravelu, S. Sreenadh, and V. R. Babu, "Peristaltic transport of a Herschel-Bulkley fluid in an inclined tube," International Journal of Non-Linear Mechanics, vol. 40, no. 1, pp. 83-90, 2005.

[6] K. S. Mekheimer and Y. Abd elmaboud, "The influence of heat transfer and magnetic field on peristaltic transport of a Newtonian fluid in a vertical annulus: application of an endoscope," Physics Letters A, vol. 372, no. 10, pp. 1657-1665, 2008.

[7] K. S. Mekheimer and Y. Abd elmaboud, "Peristaltic flow of a couple stress fluid in an annulus: application of an endoscope," Physica A, vol. 387, no. 11, pp. 2403-2415, 2008. 
[8] K. De Vries, E. A. Lyons, G. Ballard, C. S. Levi, and D. J. Lindsay, "Contractions of the inner third of the myometrium," American Journal of Obstetrics and Gynecology, vol. 162, no. 3, pp. 679-682, 1990.

[9] G. C. Shit, M. Roy, and E. Y. K. Ng, "Effect of induced magnetic field on peristaltic flow of a micropolar fluid in an asymmetric channel," International Journal for Numerical Methods in Biomedical Engineering, vol. 26, no. 11, pp. 13801403, 2010.

[10] A. Yildirim and S. A. Sezer, "Effects of partial slip on the peristaltic flow of a MHD Newtonian fluid in an asymmetric channel," Mathematical and Computer Modelling, vol. 52, no. 34, pp. 618-625, 2010.

[11] K. S. Mekheimer, S. Z. A. Husseny, and Y. Abd Elmaboud, "Effects of heat transfer and space porosity on peristaltic flow in a vertical asymmetric channel," Numerical Methods for Partial Differential Equations, vol. 26, no. 4, pp. 747-770, 2010.

[12] K. S. Mekheimer, S. Z.-A. Husseny, and A. I. Abd El Lateef, "Effect of lateral walls on peristaltic flow through an asymmetric rectangular duct," Applied Bionics and Biomechanics, vol. 8, no. 3-4, pp. 295-308, 2011.

[13] S. Srinivas and R. Muthuraj, "Effects of chemical reaction and space porosity on MHD mixed convective flow in a vertical asymmetric channel with peristalsis," Mathematical and Computer Modelling, vol. 54, no. 5-6, pp. 1213-1227, 2011.

[14] S. K. Pandey and M. K. Chaube, "Peristaltic transport of a maxwell fluid in a channel of varying cross section induced by asymmetric waves: application to embryo transport within uterine cavity," Journal of Mechanics in Medicine and Biology, vol. 11, no. 3, pp. 675-690, 2011.

[15] S. Srinivas, R. Gayathri, and M. Kothandapani, "Mixed convective heat and mass transfer in an asymmetric channel with peristalsis," Communications in Nonlinear Science and Numerical Simulation, vol. 16, no. 4, pp. 1845-1862, 2011.

[16] K. Das, "Influence of slip and heat transfer on mhd peristaltic flow of a Jeffrey fluid in an inclined asymmetric porous channel," Indian Journal of Mathematics, vol. 54, pp. 19-45, 2012.

[17] Y. Abd Elmaboud, S. Kh. Mekheimer, and A. I. Abdellateef, "Thermal properties of couple-stress fluid flow in an asymmetric channel with peristalsis," Journal of Heat Transfer, vol. 135, Article ID 044502, 2013.

[18] N. S. Akbar and S. Nadeem, "Endoscopic effects on peristaltic flow of a nanofluid," Communications in Theoretical Physics, vol. 56, no. 4, pp. 761-768, 2011.

[19] N. S. Akbar, S. Nadeem, T. Hayat, and A. A. Hendi, "Peristaltic flow of a nanofluid in a non-uniform tube," Heat and Mass Transfer, vol. 48, no. 3, pp. 451-459, 2012.

[20] N. S. Akbar and S. Nadeem, "Peristaltic flow of a Phan-ThienTanner nanofluid in a diverging tube," Heat Transfer, vol. 41, no. 1, pp. 10-22, 2012.

[21] M. Mustafa, S. Hina, T. Hayat, and A. Alsaedi, "Influence of wall properties on the peristaltic flow of a nanofluid: analytic and numerical solutions," International Journal of Heat and Mass Transfer, vol. 55, no. 17-18, pp. 4871-4877, 2012.

[22] N. S. Akbar, S. Nadeem, T. Hayat, and A. A. Hendi, "Peristaltic flow of a nanofluid with slip effects," Meccanica, vol. 47, no. 5, pp. 1283-1294, 2012.

[23] O. A. Bég and D. Tripathi, "Mathematica simulation of peristaltic pumping with double-diffusive convection in nanofluids: a bio-nano-engineering model," Journal of Nanoengineering and Nanosystems, vol. 225, no. 3, pp. 99-114, 2012.
[24] S. U. S. Choi, "Enhancing thermal conductivity of fluids with nanoparticles," in Proceedings of the ASME International Mechanical Engineering Congress and Exposition, vol. 66 of FED 231/MD, p. 99, ASME, San Francisco, Calif, USA, 1995.

[25] S. U. S. Choi, Z. G. Zhang, W. Yu, F. E. Lockwood, and E. A. Grulke, "Anomalous thermal conductivity enhancement in nanotube suspensions," Applied Physics Letters, vol. 79, no. 14, pp. 2252-2254, 2001.

[26] Q. A. Pankhurst, J. Connolly, S. K. Jones, and J. Dobson, "Applications of magnetic nanoparticles in biomedicine," Journal of Physics D, vol. 36, no. 13, pp. R167-R181, 2003.

[27] M. R. Habibi, M. Ghassemi, and M. H. Hamedi, "Analysis of high gradient magnetic field effects on distribution of nanoparticles injected into pulsatile blood stream," Journal of Magnetism and Magnetic Materials, vol. 324, no. 8, pp. 14731482, 2012.

[28] A. Ebaid and E. H. Aly, "Exact analytical solution of the peristaltic nanofluids flow in an asymmetric channel with flexible walls and slip condition: application to the cancer treatment," Computational and Mathematical Methods in Medicine, vol. 2013, Article ID 825376, 8 pages, 2013.

[29] T. Fang, S. Yao, J. Zhang, and A. Aziz, "Viscous flow over a shrinking sheet with a second order slip flow model," Communications in Nonlinear Science and Numerical Simulation, vol. 15, no. 7, pp. 1831-1842, 2010.

[30] M. M. Nandeppanavar, K. Vajravelu, M. S. Abel, and M. N. Siddalingappa, "Second order slip flow and heat transfer over a stretching sheet with non-linear Navier boundary condition," International Journal of Thermal Sciences, vol. 58, pp. 143-150, 2012.

[31] M. Turkyilmazoglu, "Heat and mass transfer of MHD second order slip flow," Computers \& Fluids, vol. 71, pp. 426-434, 2013.

[32] A. V. Rosca and I. Pop, "Flow and heat transfer over a vertical permeable stretching/shrinking sheet with a second order slip," International Journal of Heat and Mass Transfer, vol. 60, pp. 355364, 2013.

[33] E. H. Aly and A. Ebaid, "Exact analytical solution for suction and injection flow with thermal enhancement of five nanofluids over an isothermal stretching sheet with effect of the slip model: a comparative study," Abstract and Applied Analysis, vol. 2013, Article ID 721578, 14 pages, 2013.

[34] M. Mishra and A. Ramachandra Rao, "Peristaltic transport of a Newtonian fluid in an asymmetric channel," Zeitschrift fur Angewandte Mathematik und Physik, vol. 54, no. 3, pp. 532-550, 2003. 


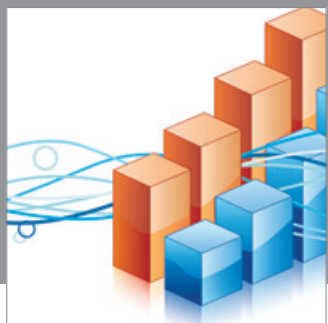

Advances in

Operations Research

mansans

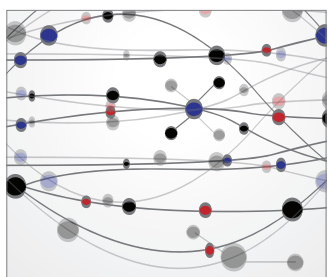

The Scientific World Journal
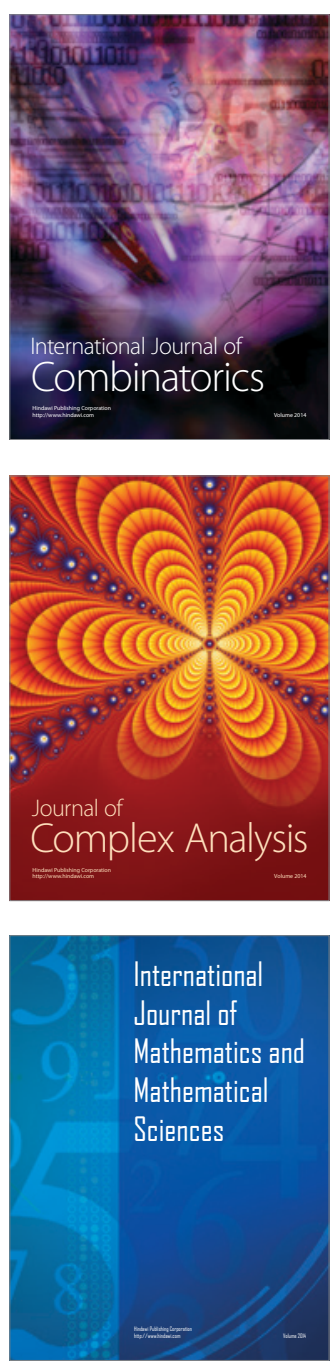
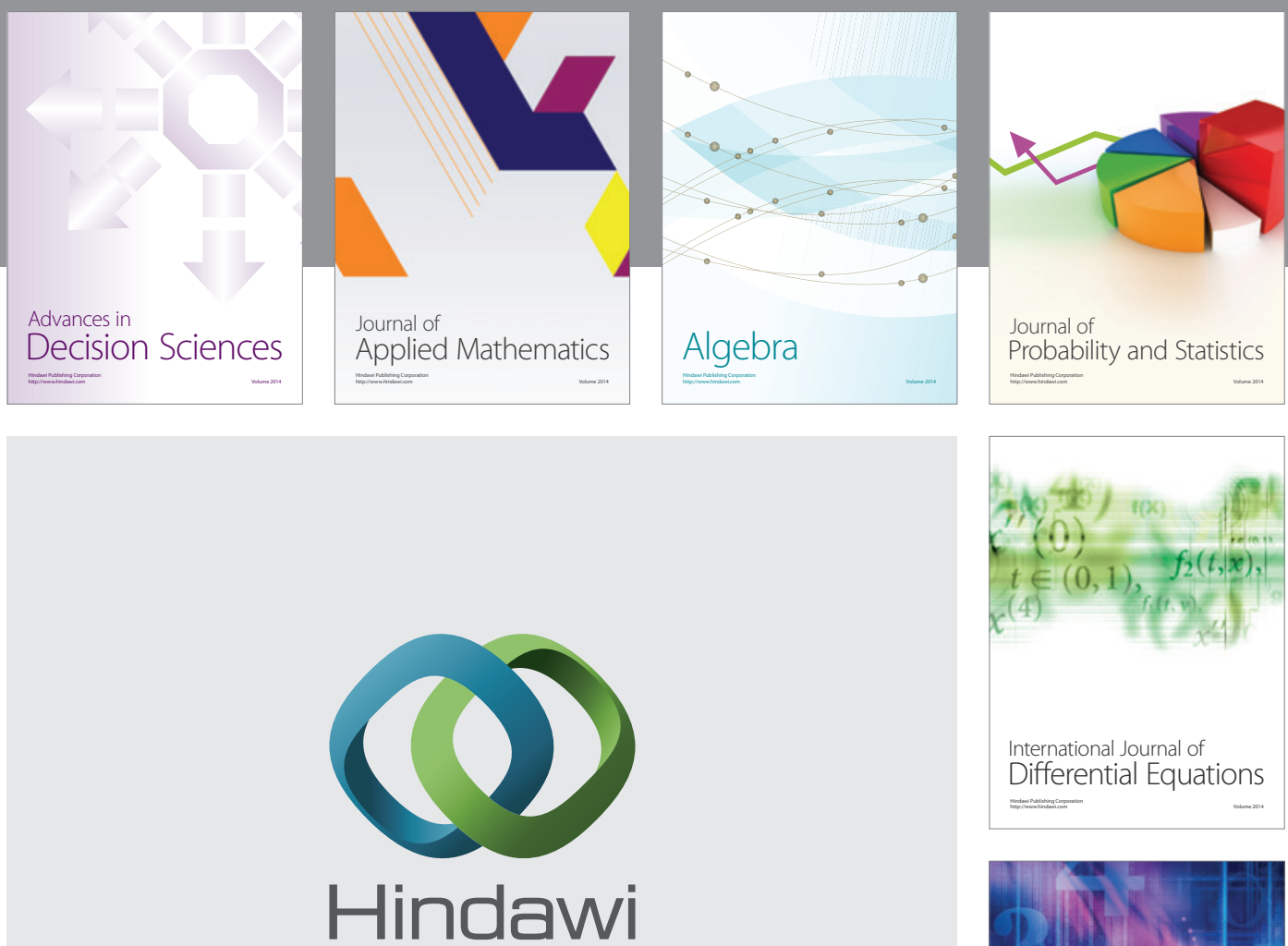

Submit your manuscripts at http://www.hindawi.com
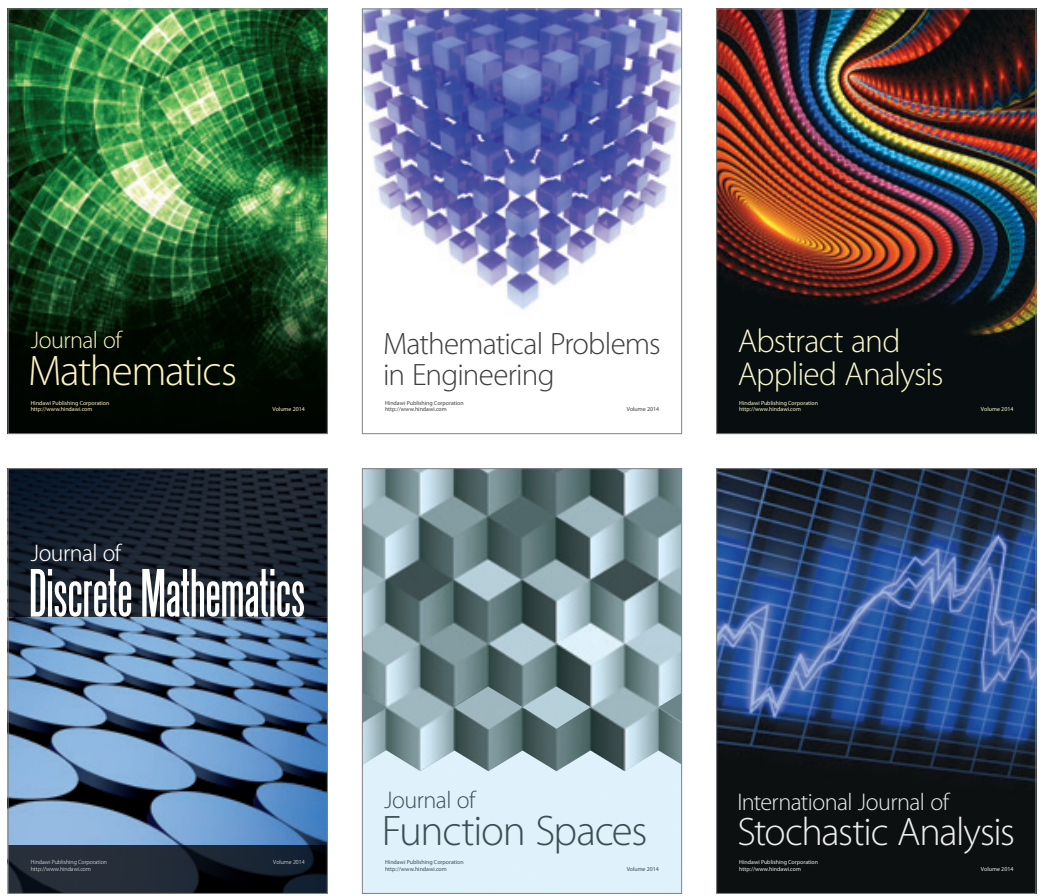

Journal of

Function Spaces

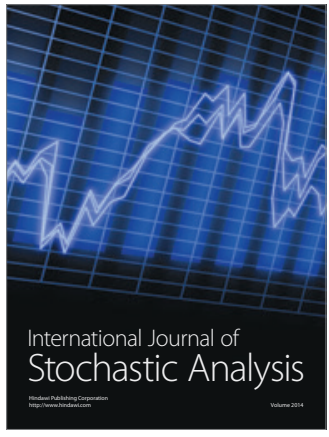

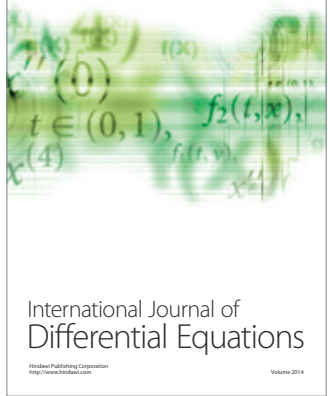
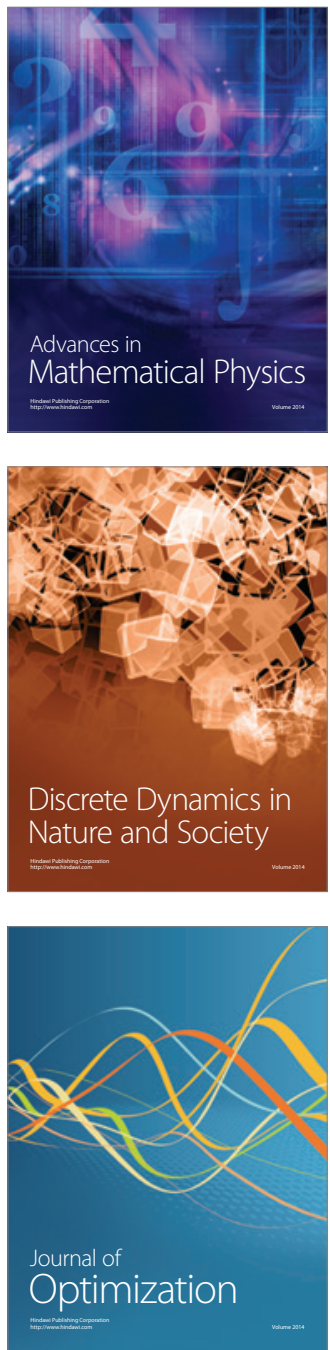